\title{
Editorial: Special issue of PODC 2017 and DISC 2017
}

Hagit Attiya ${ }^{1}$

Published online: 4 April 2020

(c) Springer-Verlag GmbH Germany, part of Springer Nature 2020

This special issue of Distributed Computing is based on papers that originally appeared as extended abstracts in the Proceedings of the 36th Symposium on Principles of distributed computing (PODC 2017), held in Washington, DC, USA, and in the Proceedings of the 31st Symposium on Principles of distributed computing (DISC 2017), held in Vienna, Austria.

The papers for the Special Issue were chosen by the respective Program Committees from the regular papers presented at the symposium, based on their quality and representation of the spectrum of topics encompassed by the Symposium. In addition to being reviewed, in preliminary form, by the respective Program Committees, the full papers submitted for the Special Issue were refereed according to the standard practices of Distributed Computing. We thank the handling editors, the referees and the authors of these papers.

Publisher's Note Springer Nature remains neutral with regard to jurisdictional claims in published maps and institutional affiliations.
Hagit Attiya

hagit@cs.technion.ac.il

1 Technion, Haifa, Israel 